\title{
COMMUTING BOOLEAN ALGEBRAS OF PROJECTIONS. II. BOUNDEDNESS IN $L_{p}$
}

\author{
C. A. MCCARTHY ${ }^{1}$
}

This paper complements our previous paper of the same title [4]. The conclusions, results applicable to the theory of spectral operators, are those of the previous paper, but the hypotheses are disjoint and the methods are somewhat different; we will make only historical reference to this work.

One of the basic problems of the theory of spectral operators is whether the sum and product of two commuting spectral operators on a Banach space is again spectral (for background material on spectral operators see especially [1] or [2]). Wermer [5] showed that this is in fact always the case if the operators act on a Hilbert space. Dunford [1] and Foguel [3] proved that if the underlying space is weakly complete, the boundedness of the Boolean algebra of projections generated by the resolutions of the identity of the operators implies that the sum and product is spectral. In practice, however, this may be difficult to determine, and our work has been to find easily applicable criteria for this boundedness. Our previous paper [4] gave a criterion in terms of multiplicity: It sufficed that one of the algebras of projections was of finite multiplicity, and that even for some separable reflexive Banach spaces this condition was necessary. Our present criterion is in terms of the underlying space and independent of various properties the generating Boolean algebras might enjoy other than their boundedness. Our result holds for $L_{p}$ spaces, $1<p$ $<\infty$, direct sums of $L_{p}$ spaces for which the $p$ 's are bounded away from 1 and $\infty$, and for subspaces thereof; especially of interest for partial differential equations is that our theorem holds for the Sobolev spaces where the norm of a function $x$ is given by the sum of the $L_{p}$ norm of $x$ and of some perhaps different $L_{p}$ norms of its derivatives. Since our estimates are all finite-combinatoric, our results hold for inseparable $L_{p}$ spaces, $L_{p}$ spaces with respect to only finitely additive measures, and their elaborations.

Our first section will establish some combinatorial propositions, which when used in the second section will give our theorem for $2 \leqq p<\infty$; consideration of adjoints gives the theorem for $1<p \leqq 2$.

Received by the editors December 29, 1962, and, in revised form, June 13, 1963 .

${ }^{1}$ Supported by NSF G-14137. 
1. Averaging propositions. We shall wish to consider the average value of such expressions as $\left|\sum_{j} c_{j} \mu_{j}\right|^{p}$ as the $c_{j}$ vary independently over complex numbers of absolute value one; to be explicit, we define such things as Average $\left.\right|_{c_{j} \mid=1}\left|\sum_{j=1}^{n} c_{j} \mu_{j}\right|^{p}$ to be

$$
(2 \pi)^{-n} \int_{0}^{2 \pi} d \theta_{1}, \cdots, \int_{0}^{2 \pi} d \theta_{n}\left|\sum_{j=1}^{n} e^{i \theta_{i}} \mu_{j}\right|^{p} \text {. }
$$

Proposition 1. Let $\mu_{1} \cdots \mu_{n}$ be any complex numbers, and $p \geqq 2$. Then

$$
\left(\sum_{j}\left|\mu_{j}\right|^{2}\right)^{p / 2} \leqq \underset{\left|c_{j}\right|=1}{\text { Average }}\left|\sum_{j} c_{j} \mu_{j}\right|^{p} \leqq \Gamma\left(\frac{p}{2}+2\right)\left(\sum_{j}\left|\mu_{j}\right|^{2}\right)^{p / 2} .
$$

Proof. First assume $p$ is an even integer, $p=2 N$. Then

$$
\begin{aligned}
\left|\sum_{j} c_{j} \mu_{j}\right|^{2 N} & =\left(\sum_{j, k} c_{j} \bar{c}_{k} \mu_{j} \bar{\mu}_{k}\right)^{N} \\
& =\sum_{\substack{j_{1}, \cdots, j_{N} \\
k_{1}, \cdots, k_{N}}} c_{j_{1}} \cdots c_{j_{N}} \bar{c}_{k_{1}} \cdots \bar{c}_{k_{N}} \mu_{j_{1}} \cdots \mu_{j_{N}} \bar{\mu}_{k_{1}} \cdots \bar{\mu}_{k_{N}} .
\end{aligned}
$$

Now when we perform the integrations to compute the average, all terms vanish except those for which $k_{1}, \cdots, k_{N}$ is a permutation of $j_{1}, \cdots, j_{N}$; and such a term contributes $\left|\mu_{j_{1}}\right|^{2} \cdots\left|\mu_{j_{N}}\right|^{2}$ to the average for each distinct permutation of the $j$ 's. If a given set $j_{1}, \cdots, j_{N}$ consists of $r$ integers with multiplicities $m_{1}, \cdots, m_{r}$, then the number of distinct permutations is the $N$-nomial coefficient $N ! / m_{1} ! m_{2} ! \cdots m_{r}$ ! which in any case lies between 1 and $N !$.

Thus the average lies between

$$
\sum_{j_{1}}, \cdots, j_{N}\left|\mu_{j_{1}}\right|^{2} \cdots\left|\mu_{j_{N}}\right|^{2}=\left(\sum\left|\mu_{j}\right|^{2}\right)^{N} \quad \text { and } N !\left(\sum_{j}\left|\mu_{j}\right|^{2}\right)^{N} \text {, }
$$

proving the proposition in this case.

For other $p$, set $p=2(N+\epsilon)$, where $N$ is an integer and $0<\epsilon<1$. Hölder's inequality, applied to the integrals defining the average, yields an upper bound:

$$
\begin{aligned}
\underset{\left|c_{j}\right|=1}{\operatorname{Average}}\left|\sum_{j} c_{j} \mu_{j}\right|^{2(N+\epsilon)} & \leqq\left(\underset{\left|c_{j}\right|=1}{\text { Average }}\left|\sum_{j} c_{j} \mu_{j}\right|^{2(N+1)}\right)^{(N+\epsilon) /(N+1)} \\
& \leqq \Gamma(N+2)\left(\sum_{j}\left|\mu_{j}\right|^{2}\right)^{N+\epsilon}
\end{aligned}
$$

and the lower bound: 


$$
\begin{aligned}
\left(\sum_{j}\left|\mu_{j}\right|^{2}\right)^{(N+\epsilon)} & \leqq\left(\underset{\left|c_{j}\right|=1}{\text { Average }}\left|\sum_{j} c_{j} \mu_{j}\right|^{2 N}\right)^{(N+\epsilon) / N} \\
& \leqq \underset{\left|c_{j}\right|=1}{\text { Average }}\left|\sum_{j} c_{j} \mu_{j}\right|^{2(N+\epsilon)} .
\end{aligned}
$$

Proposition 2. Let $\lambda_{j k}, j=1, \cdots, n, k=1, \cdots, m$ be any complex numbers and let $p \geqq 2$. Then

$$
\begin{aligned}
\left(\sum_{j k}\left|\lambda_{j k}\right|^{2}\right)^{p / 2} & \leqq \underset{\left|d_{k}\right|=1}{\operatorname{Average}}\left(\sum_{j}\left|\sum_{k} d_{k} \lambda_{j k}\right|^{2}\right)^{p / 2} \\
& \leqq \Gamma\left(\frac{p}{2}+2\right)\left(\sum_{j k}\left|\lambda_{j k}\right|^{2}\right)^{p / 2} .
\end{aligned}
$$

Proof. We first obtain the upper bound. Let $p$ be an even integer, $p=2 N$. We have

$$
\begin{aligned}
& \left(\sum_{j}\left|\sum_{k} d_{k} \lambda_{j k}\right|^{2}\right)^{N} \\
& \quad=\sum_{\substack{k_{1}, \cdots, k_{N} \\
l_{1}, \cdots, l_{N}}} d_{k_{1}} \cdots d_{k_{N}} \bar{d}_{l_{1}} \cdots \bar{d}_{l_{N}}\left(\sum_{j} \lambda_{j k_{1}} \bar{\lambda}_{j l_{1}}\right) \cdots\left(\sum_{j} \lambda_{j k_{N}} \bar{\lambda}_{j l_{N}}\right) .
\end{aligned}
$$

As before, the only nonzero contributions after performing the integrations to compute the average are those for which $l_{1}, \cdots, l_{N}$ is a permutation of $k_{1}, \cdots, k_{N}$. For any such permutation, the contribution is bounded in absolute value by

$$
\begin{aligned}
& \left|\sum_{j} \lambda_{j k_{1}} \bar{\lambda}_{j l_{1}}\right| \cdots\left|\sum_{j} \lambda_{j k_{N}} \bar{\lambda}_{j l_{N}}\right| \\
& \leqq\left(\sum_{j}\left|\lambda_{j k_{1}}\right|^{2}\right)^{1 / 2}\left(\sum_{j}\left|\lambda_{j l_{1}}\right|^{2}\right)^{1 / 2} \cdots\left(\sum_{j}\left|\lambda_{j k_{N}}\right|^{2}\right)^{1 / 2}\left(\sum_{j}\left|\lambda_{j l_{N}}\right|^{2}\right)^{1 / 2} \\
& =\left(\sum_{j}\left|\lambda_{j k_{1}}\right|^{2}\right) \cdots\left(\sum_{j}\left|\lambda_{j k_{N}}\right|^{2}\right) .
\end{aligned}
$$

Since there are at most $N$ ! such permutations, the average is bounded above by

$$
N ! \sum_{k_{1}, \cdots, k_{N}}\left(\sum_{j}\left|\lambda_{j k_{1}}\right|^{2}\right) \cdots\left(\sum_{j}\left|\lambda_{j k_{N}}\right|^{2}\right)=N !\left(\sum_{j, k}\left|\lambda_{j k}\right|^{2}\right)^{N} .
$$

The upper bound for general $p$ is obtained from the Hölder inequality exactly as in Proposition 1. 
For the lower bound, we consider first $p=2$ :

$$
\begin{aligned}
\underset{\left|d_{k}\right|=1}{\operatorname{Average}} \sum_{j}\left|\sum_{k} d_{k} \lambda_{j k}\right|^{2} & =\text { Average } \sum_{k, l} d_{k} \bar{d}_{l} \sum_{j} \lambda_{j k} \bar{\lambda}_{j l} \\
& =\sum_{k} \sum_{j} \lambda_{j k} \bar{\lambda}_{j k}=\sum_{j k}\left|\lambda_{j k}\right|^{2} .
\end{aligned}
$$

For any other $p$, we Hölder:

$$
\begin{aligned}
\left(\sum_{j, k}\left|\lambda_{j k}\right|^{2}\right)^{p / 2} & \left.=\underset{\substack{\text { Average } \\
\left|d_{k}\right|=1}}{ } \sum_{j}\left|\sum_{k} d_{k} \lambda_{j k}\right|^{2}\right)^{p / 2} \\
& \leqq \underset{\left|d_{k}\right|=1}{\operatorname{Average}}\left(\sum_{j}\left|\sum_{k} d_{k} \lambda_{j k}\right|^{2}\right)^{p / 2} .
\end{aligned}
$$

Propositions 1 and 2 together give the inequality we wish, namely.

$$
\begin{aligned}
& \left(\sum_{j, k}\left|\lambda_{j k}\right|^{2}\right)^{p / 2} \leqq \underset{\left|c_{j}\right|=1,\left|d_{k}\right|=1}{\text { Average }}\left|\sum_{j, k} c_{j} d_{k} \lambda_{j k}\right|^{p} \\
& \leqq \Gamma\left(\frac{p}{2}+2\right)^{2}\left(\sum_{j, k}\left|\lambda_{j k}\right|^{2}\right)^{p / 2}
\end{aligned}
$$

for any complex numbers $\lambda_{j k}$, and $p \geqq 2$.

2. The boundedness theorem. Let $\varepsilon$ be a Boolean algebra of projections on a Banach space. A theorem of Dunford [1, Theorem 7] states that if $E_{1}, \cdots, E_{n}$ are mutually disjoint members of $\varepsilon$, and if $a_{1}, \cdots, a_{n}$ are complex numbers, then the norm of the operator $\sum_{j=1}^{n} a_{j} E_{j}$ is at most $4 \cdot \max _{j}\left|a_{j}\right| \sup _{E \in \mathcal{E}}\|E\|$. We define $\|\mathcal{E}\|$ to be $\sup \left\{\left\|\sum_{j} a_{j} E_{j}\right\|:\left|a_{j}\right| \leqq 1, E_{j}\right.$ disjoint projections in $\left.\mathcal{E}\right\}$ and note that $\|\varepsilon\|$ is finite, if and only if there is a finite upper bound to $\|E\|, E \in \varepsilon$.

The BoUndedness THEOREM. Let $\mathcal{E}$ and $\mathfrak{F}$ be two bounded commuting Boolean algebras of projections on $L_{p}$, and let $G$ be the Boolean algebra of projections consisting of finite sums of disjoint projections $E F, E \in \mathcal{E}$, $F \in \mathcal{F}$. Then

$$
\|S\| \leqq\left(\Gamma\left(\frac{p}{2}+2\right)\right)^{2 / p}\|\mathcal{E}\| 2\|\mathcal{F}\|^{2}
$$

if $p \geqq 2$, or the same quantity with $p$ replaced by $p /(p-1)$ if $p<2$.

Proof. If $\mathcal{E}^{*}=\left\{E^{*}: E \in \varepsilon\right\}$, then the Boolean algebra $\varepsilon^{*}$ satisfies $\|\mathcal{E}\|=\left\|\mathcal{E}^{*}\right\|$, so that it suffices to consider, of $L_{p}$ and $L_{p /(p-1)}$, only that one of larger exponent.

When we compute $\|g\|$ it suffices to consider operators of the form 
$\sum_{j=1}^{n} \sum_{k=1}^{m} a_{j k} E_{j} F_{k}$ with $\left|a_{j k}\right|=1$ and $\sum_{j} E_{j}=\sum_{k} F_{k}=I$, the identity on $L_{p}$; for any operator which we use to compute $\|g\|$ is in the convex hull of such operators. Now let $x$ be a function in $L_{p}$. We wish to compute $\left\|\sum_{j k} a_{j k} E_{j} F_{k} x\right\| p$. For any choice of $c_{1}, \cdots, c_{n}$ each of absolute value 1 , the operator $\sum_{j} c_{j} E_{j}$, as well as its inverse $\sum_{j} \bar{c}_{j} E_{j}$, has bound $\|\varepsilon\|$. The analogous holds true for an operator $\sum_{k} d_{k} F_{k}$ where $d_{1}, \cdots, d_{m}$ are each of absolute value 1 . Thus

$$
\begin{aligned}
\|\varepsilon\|^{-p}\|\mathcal{F}\|^{-p}\left\|\sum_{j, k} c_{j} d_{k} a_{j k} E_{j} F_{k} x\right\|^{p} & \leqq\left\|\sum_{j, k} a_{j k} E_{j} F_{k} x\right\|^{p} \\
& \leqq\|\varepsilon\| p\|\mathcal{F}\| p\left\|\sum_{j, k} c_{j} d_{k} a_{j k} E_{j} F_{k} x\right\|^{p} .
\end{aligned}
$$

If we average over all $\left|c_{j}\right|=1,\left|d_{k}\right|=1$, the central term of this in equality remains unchanged. However,

$$
\begin{aligned}
\underset{\left|c_{j}\right|=1,\left|d_{k}\right|=1}{\text { Average }}\left\|\sum_{j, k} c_{j} d_{k} a_{j k} E_{j} F_{k} x\right\|^{p} & =\underset{\left|c_{j}\right|=1,\left|d_{k}\right|=1}{\operatorname{Average}} \int\left|\sum_{j, k} c_{j} d_{k} a_{j k} E_{j} F_{k} x\right|^{p} \\
& =\int \underset{\substack{\text { Average } \\
\left|c_{j}\right|=1,\left|d_{k}\right|=1}}{\text { Averag }}\left|\sum_{j, k} c_{j} d_{k} a_{j k} E_{j} F_{k} x\right|^{p} .
\end{aligned}
$$

By Propositions 1 and 2, this is bounded below by

$$
\int\left(\sum_{j, k}\left|a_{j k} E_{j} F_{k} x\right|^{2}\right)^{p / 2}=\int\left(\sum_{j, k}\left|E_{j} F_{k} x\right|^{2}\right)^{p / 2}
$$

(remember that $\left|a_{j k}\right|=1$ ), and above by

$$
\Gamma\left(\frac{p}{2}+2\right)^{2} \cdot \int\left(\sum_{j, k}\left|E_{j} F_{k} x\right|^{2}\right)^{p / 2} .
$$

We thus have

$$
\begin{aligned}
\left\|\sum_{j, k} a_{j k} E_{j} F_{k} x\right\|^{p} & \leqq\|\mathcal{E}\| p\|\mathfrak{F}\|^{p} \cdot \Gamma\left(\frac{p}{2}+2\right)^{2} \int\left(\sum_{j, k}\left|E_{j} F_{k} x\right|^{2}\right)^{p / 2} \\
& \leqq\|\mathcal{E}\| p\|\mathfrak{F}\| p \Gamma\left(\frac{p}{2}+2\right)^{2} \underset{\left|c_{j}\right|=1,\left|d_{k}\right|=1}{\text { Average }}\left\|\sum_{j, k} c_{j} d_{k} E_{j} F_{k} x\right\|^{p} \\
& \leqq\|\mathcal{E}\|^{2 p \|}\|\mathfrak{F}\|^{2 p} \Gamma\left(\frac{p}{2}+2\right)^{2}\|x\| p
\end{aligned}
$$

Therefore $\|\oint\| \leqq \Gamma(p / 2+2)^{2 / p}\|\varepsilon\|^{2}\|\mathcal{I}\|^{2}$.

We wish to make the remark that the only assumptions about the underlying Banach space was that it was a space of functions $x$ in 
which the norm was defined as $\left(\int|x|^{p}\right)^{1 / p}$. The $E^{\prime}$ 's and $F^{\prime}$ 's may be such that they only operate on a certain subspace, as is the case with the Sobolev spaces which can be regarded as a subspace of a sum of $L_{p}$ spaces; thus, our theorem holds in such spaces, and of course for others as mentioned in the introduction.

3. Further remarks. If $E_{1}, \cdots, E_{n}$ are the atoms of a finite Boolean algebra $\mathcal{E}$ of projections on $L_{2}$, then an equivalent norm \|\|$\cdot \|$ on $L_{2}$ is given by

$$
\|\mathcal{E}\|^{-2}\|x\|^{2} \leqq\|x \mid\|^{2}=\sum_{j}\left\|E_{j} x\right\|^{2} \leqq\|\mathcal{E}\|^{2}\|x\|^{2} .
$$

Now if $\mathcal{F}$ is a bounded commuting algebra of projections, we have

$$
\|\| \sum_{j} E_{j} F_{j} x\left\|\left.\right|^{2}=\sum_{j}\right\| E_{j} F_{j} x\left\|^{2} \leqq\right\| \mathfrak{F}\left\|^{2}\right\| x \|^{2}
$$

so that if $\mathcal{G}=\left\{\sum_{j} E_{j} F_{j} ; E_{j}\right.$ disjoint in $\left.\mathcal{E}, F_{j} \in \mathcal{F}\right\}$, then $\|\mathcal{G}\| \leqq 4\|\mathcal{E}\| 2\|\mathfrak{F}\|$. This is essentially Wermer's method. It is natural to ask whether in general $\left(\sum_{j}\left\|E_{j} x\right\|^{p}\right)^{1 / p}$ is an equivalent norm in $L_{p}$, the equivalence depending only upon $\left\|\varepsilon_{\|}\right\|$; for then the boundedness theorem would be very simple. We are indebted to $E$. $R$. Rodemich for the following counterexample in $L_{4}$ :

In $L_{4}(0,1)$ consider the functions $\phi_{j}(t)=(-1)^{a_{j}(t)}, 0<t<1$, $1 \leqq j<\infty$, where $t$ has the dyadic expansion $t=\sum_{j=1}^{\infty} a_{j}(t) 2^{-j}$ and each $a_{j}(t)$ is either 0 or 1 ( $a_{j}$ is well defined except on a set of measure 0 ; the $\phi_{j}$ are Rademacher functions). Let the operator $E_{j}$ be defined as carrying the function $x$ into $E_{j} x=\left(\int x \phi_{j}\right) \phi_{j} . \int \phi_{j} \phi_{k}=\delta_{j k}$ shows that the operators $E_{j}$ are a disjoint set of projections. Further if we compute the norm of the operator $\sum_{j=1}^{n} a_{j} E_{j}\left(\left|a_{j}\right| \leqq 1\right)$, we have, setting $b_{j}=a_{j} \int x \phi_{j}$,

$$
\begin{aligned}
\left\|\sum_{j} a_{j} E_{j} x\right\|^{4} & =\int\left|\sum_{j} b_{j} \phi_{j}\right|^{4} \\
& =\int \sum_{j, k, l, m} b_{j} b_{k} \bar{b}_{l} \bar{b}_{m} \phi_{j} \phi_{k} \phi_{l} \phi_{m} \\
& =\sum_{j}\left|b_{j}\right|^{4}+\sum_{j, k}\left(b_{j} \bar{b}_{k}\right)^{2}+2 \sum_{j, k}\left|b_{j} b_{k}\right|^{2} \\
& \leqq 4\left(\sum_{j}\left|b_{j}\right|^{2}\right)^{2} .
\end{aligned}
$$

(We have used $\int \phi_{j} \phi_{k} \phi_{l} \phi_{m}=0$ or 1 , and 1 only in the cases $j=k=l=m$, $j=k \neq l=m, j=l \neq k=m$, and $j=m \neq k=l$.) 
Since the functions $\phi_{j}$ are orthonormal in $L_{2}(0,1), \sum_{j}\left|b_{j}\right|^{2}$ $=\sum_{j}\left|\int x \phi_{j}\right|^{2} \leqq \int|x|^{2} \leqq\|x\|_{4}^{2}$; thus the norm of the operator $\sum_{j} a_{j} E_{j}$ $\left(\left|a_{j}\right| \leqq 1\right)$ is at most $4^{1 / 4}$. Therefore the Boolean algebras $\varepsilon_{n}$ whose atoms are $E_{1}, \cdots, E_{n}, E_{0}^{(n)}=I-\sum_{1}^{n} E_{j}$, satisfy $\left\|\mathcal{E}_{n}\right\|<3$, since every member of $\varepsilon_{n}$ is a finite sum of $E_{j}$ 's or $I$ minus such a finite sum.

Now let $x_{n}=\phi_{1}+\cdots+\phi_{n} . E_{j} x_{n}=\phi_{j}(0<j \leqq n)$, and $E_{j} x_{n}=0(j>n)$. The $L_{4}$ norm of $x_{n}$ has its fourth power equal to $n+3 n^{2}$. However, if $E_{j}$ runs over all atoms of $\varepsilon_{n}, \sum_{j}\left\|E_{j} x_{n}\right\|^{4}=\sum_{j=1}^{n}\left\|E_{j} x_{n}\right\|^{4}=\sum_{j=1}^{n}\left\|\phi_{j}\right\|^{4}$ $=n$ which is not comparable with $\left\|x_{n}\right\|^{4}$ uniformly in $n$.

\section{BiBLIOGRAPHY}

1. N. Dunford, Spectral operators, Pacific J. Math. 4 (1954), 321-354.

2. - A survey of the theory of spectral operators, Bull. Amer. Math. Soc. 64 (1958), 217-274.

3. S. Foguel, Sums and products of commuting spectral operators, Ark. Mat. 3 (1957), 449-461.

4. C. McCarthy, Commuting Boolean algebras of projections, Pacific J. Math. 11 (1961), 295-307.

5. J. Wermer, Commuting spectral measures on Hilbert space, Pacific J. Math. 4 (1954), 355-362.

UnIVERSITY OF MinNESOTA 